\title{
FAZO : un programme d'analyse des spectres de thermoluminescence par la méthode de déconvolution
}

\author{
F.Z. SEGHOUR*, D. MEDHAH*, A. SEGHOUR*
}

(Manuscrit refu le 29 septembre 1997, révisé le 15 novembre 1997, accepté le 12 décembre 1997)

RÉSUMÉ FAZO est un programme informatique de déconvolution des spectres de thermoluminescence, fondé sur l'algorithme de minimisation de fonctions de Levenberg-Marquardt avec les modifications apportées par Fletcher. Élaboré et controlé au sein du laboratoire de dosimétrie externe du Centre de radioprotection et de sûreté (CRS, Alger, Algérie), le programme FAZO est actuellement utilisé pour la surveillance radiologique des personnels et de l'environnement à l'aide de détecteurs thermoluminescents. Dans ce travail, sont présentées les performances de FAZO validées à l'aide de spectres de thermoluminescence simulés obtenus à partir des équations cinétiques de Randali et Wilkins ainsi que par une confrontation avec les résultats d'autres programmes élaborés dans des laboratoires ayant participé à une campagne d'intercomparaison des programmes d'analyse des spectres de thermoluminescence. Il est aussi présenté des exemples de déconvolution des spectres expérimentaux du détecteur LiF:Mg, Ti (TLD 100 et TLD 700) irradié à $10 \mathrm{mGy}$ et $20 \mathrm{~Gy}$ et du détecteur $\mathrm{CaF}_{2}: \mathrm{Dy}$ (TLD 200) exposé à $10 \mathrm{mGy}$. Enfin, les résulats obtenus par FAZO pour l'évaluation des paramètres cinétiques, l'énergie d'activation $E$ et le facteur de fréquence $s$, du matériau $\mathrm{LiF}: \mathrm{Mg}$, Ti sont en bon accord avec les valeurs les plus répandues dans la littérature consacrée à ce sujet.

ABSTRACT FAZO: A thermoluminescence glow curve analysis program by the deconvolution method.

FAZO is a computer program of thermoluminescence glow curve deconvolution based on the modified Fletcher's minimization aigorithm of LevenbergMarquardt. The FAZO program which has been elaborated and tested in the external dosimetry laboratory of the Radiation Protection and Safety Center (CRS, Algiers, Algéria) is now used for the dosimetric survey of the personnel and the gamma outdoor by means of thermoluminescent detectors. In this work, the presented performances of FAZO have been validated using simulated thermoluminescence glow curves obtained by the kinetic equation of Randall and Wilkins, and by a confrontation with the results of other programs elaborated by laboratories which have participated to a thermoluminescence

\footnotetext{
* Centre de radioprotection et de sûreté, Département de dosimétrie des rayonnements, Laboratoire de dosimétrie exteme. 02, Bd Frantz Fanon, BP 399, Alger-Gare, 16000 Alger, Algérie
} 
glow curve analysis computer programs intercomparison. Deconvolution examples of experimental glow curves of LiF:Mg, Ti (TLD 100 and TLD 700) detector irradiated at $10 \mathrm{mGy}$ and $20 \mathrm{~Gy}$ and $\mathrm{CaF}_{2}: \mathrm{Dy}$ (TLD 200) detector exposed to $10 \mathrm{mGy}$, are also presented in this paper. Finally, the obtained results concerning the kinetic parameters evaluation by FAZO, the activation energy $E$ and the frequency factor $s$, for LiF:Mg, Ti material are found to be in good agreement with most values published on this subject.

\section{Introduction}

Le suivi dosimétrique du personnel travaillant dans le domaine de la médecine nucléaire, principalement sous son aspect de la surveillance des extrémités, ainsi que le contrôle de la radioactivité ambiante autour des installations nucléaires est assuré, depuis presque cinq années, en routine, par le laboratoire de dosimétrie externe du centre de radioprotection et de sûreté d'Alger, à l'aide de détecteurs thermoluminescents. De par le passé, et dans un souci de parfaire la qualité des mesures, notamment pour les faibles doses, plusieurs projets de développement ont porté sur l'amélioration de la surveillance radiologique à l'aide des détecteurs thermoluminescents (Mebhah et al., 1996). De l'expérience acquise au cours de ces travaux, il est apparu que l'évaluation de la dose pourrait être nettement améliorée par des techniques numériques de traitement des spectres de thermoluminescence; aussi un programme informatique de déconvolution des spectres de thermoluminescence (FAZO) a-t-il été élaboré par notre équipe de recherche. En effet, la déconvolution d'un spectre en son nombre exact de pics constituants permet la mise en évidence de l'apparition de certains pics d'intérêt. De plus, l'évaluation de l'aire sous un pic d'intérêt qui est directement reliée à la dose, est d'autant plus précise que ce pic est nettement isolé des pics avoisinants. Ces deux points sont d'importance pour l'estimation des doses pour les expositions à faibles doses et des expositions dans des champs mixtes, $(\mathrm{n}-\gamma)$ principalement.

Dans cet article, sont exposées les performances du programme FAZO, qui ont été validées à l'aide de spectres de thermoluminescence simulés obtenus à partir des équations de Randall et Wilkins d'une part, ainsi qu'à l'aide de spectres du détecteur $\mathrm{LiF}: \mathrm{Mg}$, Ti utilisés lors d'une campagne d'intercomparaison des programmes d'analyse des spectres de thermoluminescence, d'autre part.

Des applications de FAZO pour la déconvolution des spectres expérimentaux des détecteurs LiF:Mg, Ti (TLD 100 et 700) et $\mathrm{CaF}_{2}:$ Dy (TLD 200), montrent son adaptabilité à l'utilisation en routine pour la dosimétrie individuelle et le contrôle du rayonnement gamma ambiant. Enfin, et en plus du facteur d'appréciation de la reconstitution du spectre exprimé par le facteur de mérite (défini plus loin), le programme FAZO donne accès aux paramètres cinétiques, énergie d'activation et facteur de fréquence, pour chaque pic du spectre, permettant ainsi une analyse plus poussée des caractéristiques microscopiques des matériaux thermoluminescents. 


\section{Modélisation du spectre de thermoluminescence}

L'énergie cédée lors du passage des rayonnements directement ionisants $(\alpha, \beta \ldots)$ ou indirectement ionisants $(X, \gamma$, neutrons) dans un détecteur thermoluminescent, induit une augmentation de la population des porteurs de charges (électrons ou trous) dans des niveaux pièges crées dans la bande interdite du réseau cristallin par la présence de défauts ou d'impuretés. Libérés généralement sous l'action d'une excitation thermique, les porteurs de charges se recombinent et restituent une fraction de l'énergie absorbée par émission de photons (luminescence). La combinaison des variations de la probabilité de dépiégeage et celle de la population des porteurs de charges dans un niveau piège en fonction de la température, assigne à l'intensité de thermoluminescence (TL) une forme de pic asymétrique. Pendant l'exposition aux rayonnements, les niveaux pièges situés à des profondeurs différentes sont peuplés en fonction des doses. Ainsi, lors de l'excitation thermique pour la liberation des porteurs de charges piégés, l'intensité de thermoluminescence prend l'aspect d'un spectre constitué d'un nombre plus ou moins important de pics correspondant au nombre de niveaux pièges vidés (Figs. la et $1 \mathrm{~b}$ ).
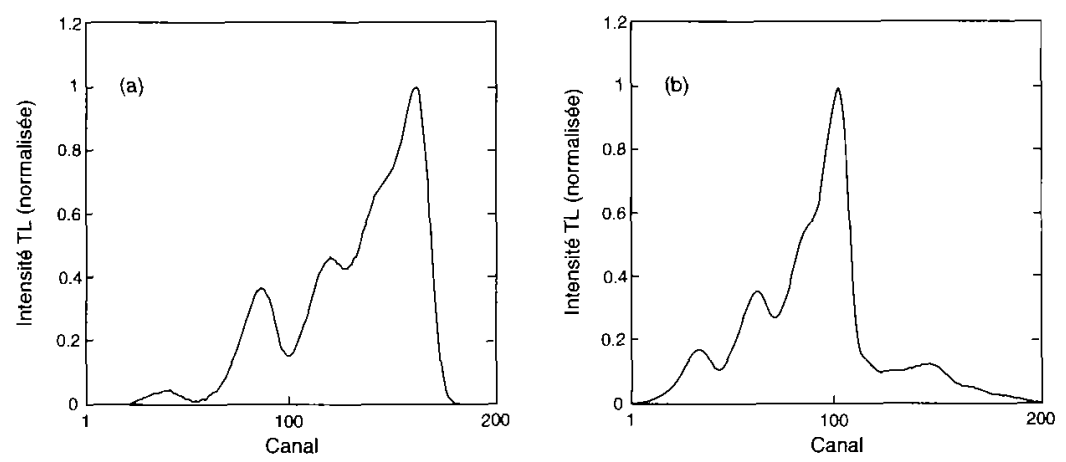

Fig. 1. - Spectres de l'émission de thermoluminescence du LiF:Mg; Ti : (a) spectre à 5 pics pour une dose de $10 \mathrm{mGy}$; (b) spectre à 9 pics pour une dose de $20 \mathrm{~Gy}$.

Thermoluminescence (TL) glow curves of LiF:Mg; Ti : (a) TL glow curve with 5 peaks for a dose of $10 \mathrm{mGy}$; (b) TL glow curve with 9 peaks for a dose of $20 \mathrm{~Gy}$.

Une modélisation mathématique des spectres de thermoluminescence peut être donnée par la fonction $S_{\mathrm{p}}(t, \mathbf{x})$ qui s'écrit sous la forme (Dehimi, 1995) :

$$
S_{\mathrm{p}}(t, \mathbf{x})=\sum_{j=1}^{N_{\mathrm{p}}} I_{j}(t, \mathbf{p})+C+I R(t)
$$


où :

$t$ : variable représentant le temps, la température ou le numéro du canal; $\mathbf{x}$ : vecteur contenant l'ensemble des paramètres cinétiques du spectre; $N_{\mathrm{p}}$ : nombre de pics constituant le spectre;

$I_{j}(t, \mathbf{p})$ : intensité de thermoluminescence du pic $j$;

$\mathbf{p}$ : vecteur des paramètres cinétiques relatifs au pic $j$;

$C$ : valeur moyenne du bruit de fond constant;

$I R(t)$ : fonction modélisant le signal infrarouge prise sous la forme:

$$
I R(t)=a \exp (-b / t) .
$$

Les expressions du signal infrarouge représenté par la fonction IR ainsi que le bruit de fond constant $C$, ne font apparaître aucun paramètre relatif à l'émission de thermoluminescence. Il est donc judicieux de soustraire leur contribution à l'expression de $S$, ce qui permet de réduire l'espace mémoire alloué à la procédure de minimisation. De plus, cette soustraction évite d'alourdir inutilement les opérations numériques que la fonction $S$ est amenée à subir lors du déroulement de l'algorithme de minimisation. De ce fait, l'équation (1) est réduite à la forme :

$$
S_{\mathrm{p}}(t, \mathbf{x})=\sum_{j=1}^{N_{\mathrm{p}}} I_{j}(t, \mathbf{p})
$$

L'expression de $I(t, \mathbf{p})$ découle de la résolution du système d'équations couplées régissant les concentrations des électrons et trous piégés en se fondant sur des modèles décrivant les phénomènes de recombinaison et de dépiégeage (Dehimi, 1995). Dans ce travail, le modèle de Randall et Wilkins (1945) dans lequel les taux de recombinaison et de dépiégeage sont supposés égaux, a été adopté et conduit à l'expression suivante :

$$
I(t, \mathbf{p})=s n_{0} \exp (-E / k T(t)) \exp \left\{-s \int_{t_{0}}^{t} \exp \left(-E / k T\left(t^{\prime}\right)\right) \mathrm{d} t^{\prime}\right\}
$$

où :

$n_{0}$ : concentration initiale des charges piégées $\left[\mathrm{m}^{-3}\right]$;

$s$ : facteur de fréquence $\left[\mathrm{s}^{-1}\right]$;

$E$ : énergie d'activation $[\mathrm{eV}]$;

$T$ : température absolue $[\mathrm{K}]$;

$k$ : constante de Boltzmann $[\mathrm{eV} / \mathrm{K}]$.

Les paramètres $E$, $s$ et $n_{0}$ apparaissant dans l'expression de $I(t, \mathbf{p})$ déterminent de façon univoque la fonction $S$, donc le spectre de thermoluminescence. 
L'énergie d'activation $E$ peut être estimée à l'aide des méthodes semi-empiriques basées sur l'analyse partielle du spectre en fonction de l'intensité maximale (Mc Keever, 1985) ou de la position du pic (Chen, 1994 ; Mc Keever, 1985) ou enfin à l'aide de la méthode de variation de la vitesse de chauffage (Chen, 1994). Toutefois, la décomposition du spectre en ses pics constituants, opération mathématique inverse de la convolution, communément appelée déconvolution (Jansson, 1984) permet à la fois l'analyse du spectre en entier et l'analyse des pics d'intérêt dosimétrique avec plus de précision.

\section{Méthode de déconvolution}

Le logiciel FAZO est élaboré à partir de l'algorithme de minimisation de fonctions de Levenberg-Marquardt qualifié de méthode à convergence rapide et précise (Fletcher, 1971). Comme pour la plupart des algorithmes de minimisation, la méthode de Levenberg-Marquardt est une méthode de minimisation au sens des moindres carrés pour laquelle la somme $Q(\mathbf{x})$ des carrés de fonctions non linéaires $r_{i}(\mathbf{x})$ à plusieurs variables $x_{i}$ est minimale pour le vecteur des paramètres cinétiques optimaux $\mathbf{x}^{*}$ (Press et al., 1988).

La fonction à minimiser $Q(\mathbf{x})$ est écrite sous la forme :

$$
Q(\mathbf{x})=\sum_{i=1}^{N}\left[r_{i}(\mathbf{x})\right]^{2}
$$

Les fonctions non linéaires $r_{i}(\mathbf{x})$, ou résidus, sont construites à partir des différences entre les intensités de thermoluminescence mesurées $y_{i}$ et celles prédites par une fonction mathématique adéquate $S_{\mathrm{p}}\left(t_{i}, \mathbf{x}\right)$ du modèle cinétique choisi. Les résidus sont définis par:

$$
r_{i}(\mathbf{x})=\underline{y}_{i}-S_{\mathrm{p}}\left(t_{i}, \mathbf{x}\right) \quad i=\overline{1, N} .
$$

Des détails sur la méthode de Levenberg-Marquardt (LM) (dite aussi méthode de Marquardt) et sur son algorithme peuvent être trouvés dans les références (Nash, 1990; Press et al., 1988). Par ailleurs, les quantités et les expressions apparaissant à chaque étape de l'algorithme sont explicitées dans la référence (Dehimi, 1995). Il est toutefois instructif pour la clarté de cet article de rappeler les étapes essentielles de l'algorithme.

Le programme de minimisation de la quantité $Q$ par rapport au vecteur des paramètres $\mathbf{x}$ suit une démarche itérative selon l'algorithme suivant:

1. Étant donné un vecteur initial $\mathbf{x}, Q(\mathbf{x})$ est évaluée à l'aide des équations (2) et (4); 
2. Un nouveau vecteur des paramètres $\mathbf{x}^{(k+1)}=\mathbf{x}^{(k)}+\delta^{(k)}$ est calculé où le vecteur $\boldsymbol{\delta}^{(k)}$ est solution de l'équation :

$$
\left(A^{(k)}+\lambda D\right) \delta^{(k)}=-\mathbf{b}^{(k)}
$$

avec $A^{(k)}$ la matrice dont l'expression est donnée par :

$$
A^{(k)}=J^{(k) \mathrm{T}} J^{(k)}
$$

et $J^{(k)}$ est le Jacobien de $\mathbf{r}$ (vecteur des résidus) :

$$
J_{i j}{ }^{(k)}=\frac{\mathrm{d} r_{i}^{(k)}}{\mathrm{d} x_{j}^{(k)}} .
$$

La matrice $D$ peut être prise comme la matrice diagonale de $A^{(k)}$ ou simplement la matrice identité. $J^{\mathrm{T}}$ est la matrice transposée de $J$.

le vecteur $\mathbf{b}^{(k)}$ est défini par:

$$
\mathbf{b}^{(k)}=J^{(k) \mathrm{T}} \mathbf{r}^{(k)} .
$$

3. Calculer $Q\left(\mathbf{x}^{(k+1)}\right)$ et comparer à $Q\left(\mathbf{x}^{(k)}\right)$ :

- si $Q\left(\mathbf{x}^{(k+1)}\right)<Q\left(\mathbf{x}^{(k)}\right)$ : l'itération a progressé en direction de la solution. Le nouveau vecteur des paramètres est pris en $\mathbf{x}^{(k+1)}$ et le processus itératif est relancé en utilisant un pas plus petit, $\lambda^{\prime}=\lambda v$;

- si $Q\left(\mathbf{x}^{(k+1)}\right)>Q\left(\mathbf{x}^{(k)}\right)$ : l'itération a progressé loin de la solution. L'actuel vecteur des paramètres $\mathbf{x}^{(k)}$ est conservé et un large pas est utilisé, $\lambda^{\prime}=\lambda v$.

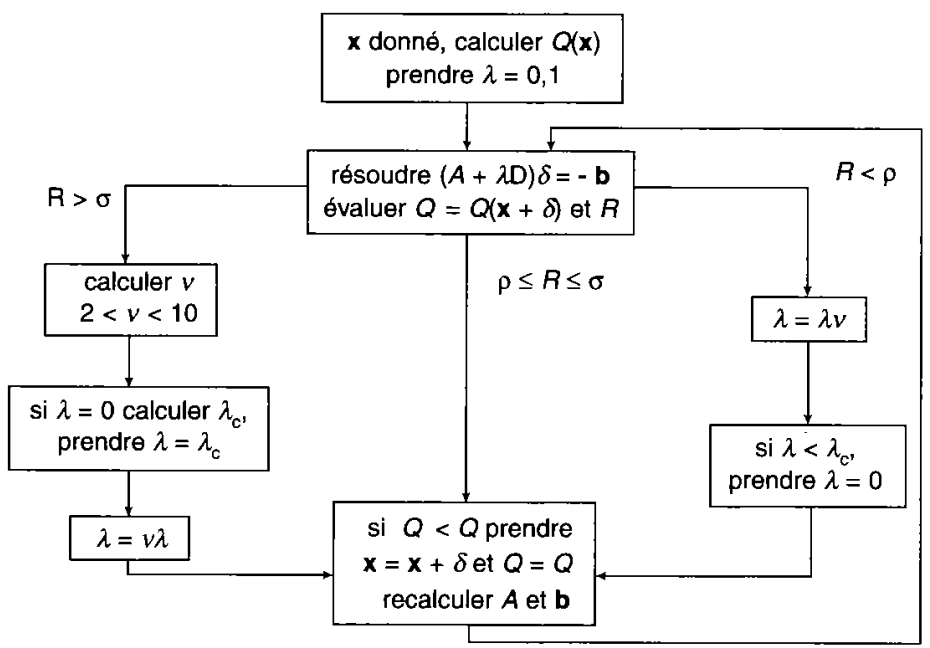

Fig. 2. - Organigramme de la procédure de Fletcher.

Fletcher's procedure organigram. 
Le paramètre apparaissant dans l'équation (6) a été initialement introduit par Marquardt afin d'assurer une convergence rapide comparable à celle de la méthode de Gauss - Newton en adoptant la stratégie de la descente selon la direction du gradient. À chaque itération, la procédure de minimisation de Marquardt, attribue à $\lambda$ des valeurs en progression géométrique croissante lorsque la fonction $S$ est loin de la solution et en progression géométrique décroissante au voisinage de la solution avec un pas, ou raison, constant. Cependant, on peut remarquer qu'en gardant un pas d'incrémentation constant,il arrive les situations de basculement perpétuel autour de la solution sans jamais l'atteindre. Pour cette raison, Fletcher (1971) apporta des modifications à l'algorithme de minimisation de Marquardt portant principalement sur la procédure d'attribution des valeurs à $\lambda$ au cours du déroulement du programme (Fig. 2). Il y introduisit un paramètre d'appréciation de la tendance de convergence sous la forme d'un rapport $R$, défini par l'expression :

$$
R=\left|\frac{Q\left(\mathbf{x}^{(k)}\right)-Q\left(\mathbf{x}^{(k+1)}\right)}{-2 \delta^{(k) \mathrm{T}} \mathbf{b}^{(k)}-\delta^{(k) \mathrm{T}} A^{(k)} \delta^{(k)}}\right| .
$$

Il a aussi défini un facteur $v$, représentant l'incrément de convergence, qui est donné par la relation :

$$
v=\frac{1}{2}\left[2-\frac{Q\left(\mathbf{x}^{(k)}\right)+\delta(\mathbf{k})-Q\left(\mathbf{x}^{(k)}\right)}{\delta^{(k) \mathrm{T}} \mathbf{b}^{(k)}}\right] .
$$

Le calcul des paramètres $R$ et $v$ conditionne le choix de la valeur affectée à $\lambda$ et permet de suivre a chaque itération l'état d'évolution du système. Cette procédure constitue le grand avantage de l'algorithme de Fletcher. Elle garantit la recherche du minimum avec plus de certitude.

Ainsi, au voisinage de la solution, $R$ est proche de 1 et le pas d'incrémentation $\delta$ doit être réduit en augmentant $\lambda$ d'un facteur $v$ pour approcher la solution avec une meilleure précision. Loin de la solution, $R$ est proche de 0 et le pas d'incrémentation $\delta$ doit être augmentée en augmentant $\lambda$ d'un facteur $1 / v$ afin de rejoindre rapidement le voisinage de la solution.

L'architecture du programme FAZO fait aussi appel au facteur de mérite (FOM : de l'anglais Figure Of Merit) comme critère d'arrêt des itérations défini par la relation (Balian et Eddy, 1977):

$$
\operatorname{FOM}(\%)=100 \times \sum_{i=1}^{N} \frac{\left|y_{i}-S_{\mathrm{p}}\left(t_{i}, \mathbf{x}\right)\right|}{A} .
$$

où :

$y_{i}$ : intensité mesurée d'un canal $i$;

$S_{\mathrm{p}}\left(t_{i}, \mathbf{x}\right)$ : intensité calculée par la fonction de modélisation du spectre;

$A$ : aire sous le spectre. 
La normalisation par rapport à l'aire du spectre apparaissant dans l'expression du FOM, rend ce dernier indépendant du spectre analysé et permet une bonne appréciation des paramètres estimés. Par ailleurs, ce facteur d'arrêt sert aussi de critère de comparaison des solutions obtenues par des programmes différents pour un même spectre. Il est communément admis qu'un facteur de mérite inférieur à $4 \%$ représente une reconstitution acceptable des spectres de thermoluminescence (Harshaw/Filtrol, 1988).

\section{Validation du logiciel FAZO}

En plus du facteur de mérite, les paramètres cinétiques des pics constituent un excellent indice d'évaluation des performances d'un programme de déconvolution d'un spectre de thermoluminescence. En effet, il est connu en déconvolution ou plus généralement dans le domaine de minimisation de fonctions, qu'une solution mathématique pour un problème physique donné peut ne pas avoir de signification physique comme pourrait être le cas d'une solution représentant une intensité ou un flux négatifs. Ainsi, et bien qu'une fonction $S$ puisse reconstituer le spectre en satisfaisant l'équation (4), il peut arriver qu'elle ne reproduise pas une solution physiquement acceptable. Ces cas sont rencontrés dans des situations où un ou plusieurs pics présentent des paramètres cinétiques $E$ ou $s$ négatifs.

L'algorithme de Levenberg-Marquardt, en sa version modifiée par Fletcher, a servi de base pour le développement du logiciel interactif FAZO de déconvolution des spectres de thermoluminescence (Dehimi, 1995 ; Seghour, 1997).

En premier, le programme FAZO a été validé à l'aide d'un spectre simulé à cinq pics, représenté en pointillés sur la figure 3 , construit à partir de 1'équation cinétique de Randall et Wilkins. L'application de FAZO pour la déconvolution de ce spectre a abouti à une restitution très satisfaisante des paramètres cinétiques $E$ et $s$ de chacun des cinq pics et avec un facteur de mérite très faible $0,38 \%$ (en trait continu sur la Fig. 3 ).

Des validations de FAZO en présence de cas réels de spectres de thermoluminescence des détecteurs LiF:Mg, Ti (TLD 100 et TLD 700) et $\mathrm{CaF}_{2}: \mathrm{Dy}$ (TLD 200), sous forme frittée de dimensions $3,1 \times 3,1 \times 0,89 \mathrm{~mm}^{3}$, utilisés en dosimétrie du personnel et de l'environnement ont été entreprises. Les conditions expérimentales de régénération et de lecture sont données dans le tableau I. Les irradiations ont été effectuées au laboratoire d'étalonnage secondaire du CRS à l'aide de sources de rayonnement gamma ${ }^{137} \mathrm{Cs}$ et ${ }^{60} \mathrm{Co}$. 


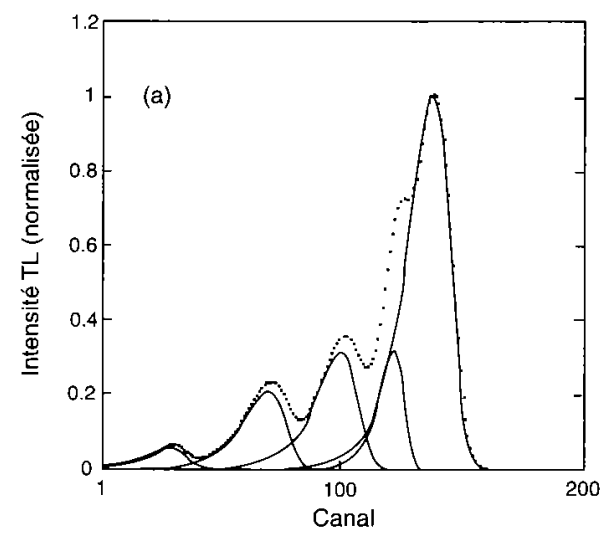

Fig. 3. - Déconvolution d'un spectre simulé de l'émission de thermoluminescence obtenu à l'aide de l'équation cinétique de Randall et Wilkins en ses 5 pics constituants.

Deconvolution of simulated TL glow curve obtained by the kinetic equation of Randall and Wilkins in its 5 constituent peaks.

\section{TABLEAU I}

Conditions expérimentales de régénération et de lecture.

Experimental conditions of thermal treatment and readout.

\begin{tabular}{|c|c|c|c|}
\hline speetre & $\begin{array}{l}\text { Cjcle } \\
\text { de régenfration }\end{array}$ & $\begin{array}{l}\text { Dose } \\
(\mathbf{G y})\end{array}$ & $\begin{array}{l}\text { de ehauffoge } \\
(\mathbf{K} / \mathbf{s})\end{array}$ \\
\hline \multirow{2}{*}{ Fig. 4 a } & & $1 \times 10^{-2}$ & 5 \\
\hline & $\begin{array}{l}1 \mathrm{~h} \text { à } 400^{\circ} \mathrm{C} \text { suivi } \\
\text { de } 2 \mathrm{~h} \text { à } 100^{\circ} \mathrm{C}\end{array}$ & & \\
\hline Fig. $4 \mathrm{~b}$ & & 20 & 5 \\
\hline Fig. 5 & $1 \mathrm{~h}$ a $400^{\circ} \mathrm{C}$ & $1 \times 10^{-2}$ & 5 \\
\hline Fig. $6 \mathrm{a}$ & $\begin{array}{l}\text { régénération } \\
\text { dans le lecteur }\end{array}$ & $1 \times 10^{-2}$ & 6 \\
\hline & $\begin{array}{l}1 \mathrm{~h} \mathrm{à} 400^{\circ} \mathrm{C} \text { suivi d'un } \\
\text { refroidissement rapide }\end{array}$ & & \\
\hline Fig. $6 \mathrm{~b}$ & $\begin{array}{l}\text { à la température ambiante, } \\
\text { et stockage à } 45^{\circ} \mathrm{C} \\
\text { pendant } 7 \text { jours }\end{array}$ & $1 \times 10^{-2}$ & 6 \\
\hline Fig. 7 a & $\begin{array}{l}\text { régénération } \\
\text { dans le lecteur }\end{array}$ & $2 \times 10^{-4}$ & 6 \\
\hline Fig. $7 \mathrm{~b}$ & $\begin{array}{l}1 \mathrm{~h} \text { à } 400^{\circ} \mathrm{C} \text { suivi d'un } \\
\text { refroidissement rapide } \\
\text { à la température ambiante }\end{array}$ & 60 & 1 \\
\hline
\end{tabular}


Pour le détecteur $\mathrm{LiF}: \mathrm{Mg}$, Ti, deux exemples de déconvolution sont donnés pour le cas d'une exposition à une faible dose de $10 \mathrm{mGy}$ (Fig. 4a) et a une forte dose de $20 \mathrm{~Gy}$ (Fig. 4b). Ces deux spectres ont été déconvolués en 4 pics pour le premier et en 9 pics pour le second; ils ont abouti à des facteurs de mérite respectifs valants $1,65 \%$ et $1,03 \%$.
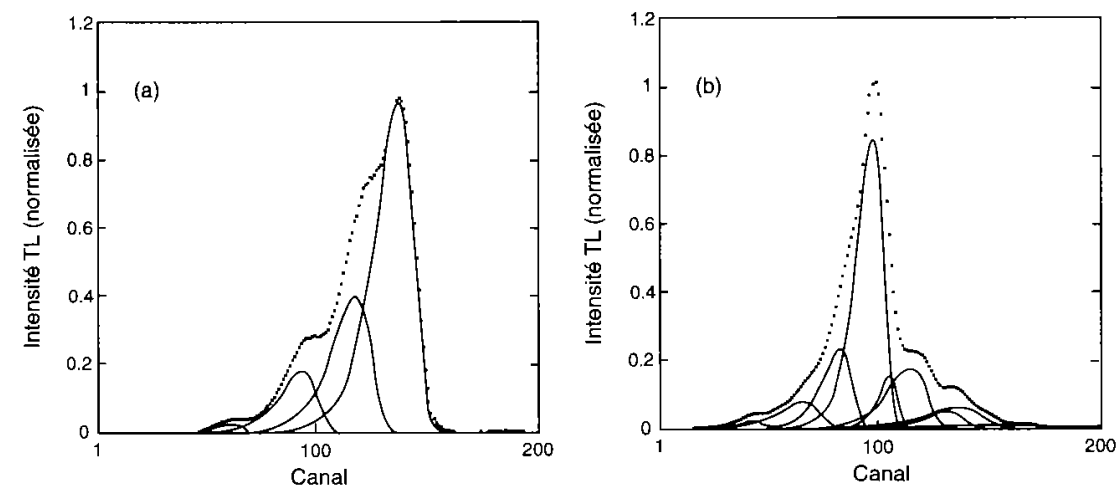

Fig. 4. - Déconvolution des spectres de LiF:Mg; Ti: (a) en 4 pics pour une dose de $10 \mathrm{mGy}$; (b) en 9 pics pour une dose de $20 \mathrm{~Gy}$.

Deconvolution of LiF:Mg; Ti glow curves : (a) in 4 peaks for a dose of $10 \mathrm{mGy}$; (b) in 9 peaks for a dose of $20 \mathrm{~Gy}$.

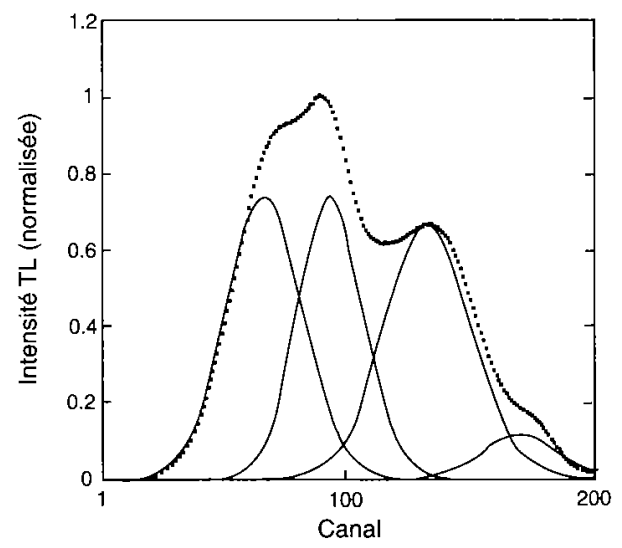

Fig. 5. - Déconvolution d'un spectre du $\mathrm{CaF}_{2}: D y$ en quatre pics pour une dose de $10 \mathrm{mGy}$. Deconvolution of $\mathrm{CaF}_{2}:$ Dy glow curve in 4 peaks for a dose of $10 \mathrm{mGy}$. 
Il est utile de signaler que l'appréciation, de la part de l'utilisateur, du nombre de pics dont est constitué le spectre, reste, pour ce type de détecteurs, un simple exercice de reconnaissance des changements de l'allure des flancs des pics les plus intenses.

Quant au détecteur $\mathrm{CaF}_{2}$ :Dy, l'estimation du nombre de pics constituant le spectre est tributaire de l'expérience de l'utilisateur habitué à manipuler ce type de spectres, en raison du chevauchement de ses pics larges et rapprochés. Ainsi, et vu la caractéristique particulière de ce spectre, un nombre important de pics qui conduirait à un FOM faible n'implique pas nécessairement une bonne déconvolution ou restitution du spectre. En effet, il a été trouvé que pour un spectre de $\mathrm{CaF}_{2}:$ Dy irradié à $10 \mathrm{mGy}$ (Fig. 5), une décomposition spectrale, et selon une distribution gaussienne, à 4 pics conduisait à un FOM de 2,63\% alors que pour 6 pics le FOM était de 0,49\% (Dehimi, 1995) encore que ce spectre ne puisse présenter que 4 pics en raison de la faible dose (Portal, 1981). Ce problème est commun à toutes les procédures interactives de déconvolution et demande une attention particulière de la part de l'utilisateur.

\section{TABLEAU II}

Liste des procédures de minimisation utilisées par les laboratoires ayant participé à l'intercomparaison GLOCANIN.

\section{List of the minimization procedures of the GLOCANIN intercomparison participating laboratories.}

\begin{tabular}{|c|c|}
\hline Arogr inner & Procture de wintwithon \\
\hline $\mathbf{A}$ & Algorithme de Levenberg-Marquardt \\
\hline $\mathbf{B}$ & $\begin{array}{l}\text { Algorithme de Levenberg-Marquardt } \\
\text { avec les modifications de Fletcher }\end{array}$ \\
\hline $\mathbf{C}$ & $\begin{array}{l}\text { Une combinaison entre l'algorithme de Newton-Raphson, de } \\
\text { Marquardt et celui de Steepest }\end{array}$ \\
\hline $\mathbf{D}$ & Algorithme de Marquardt \\
\hline $\mathbf{E}$ & $\begin{array}{l}\text { Algorithme de Levenberg-Marquardt } \\
\text { avec les modifications de Nash }\end{array}$ \\
\hline $\mathbf{F}$ & Algorithme de Marquardt \\
\hline $\mathbf{G}$ & Algorithme de Levenberg-Marquardt \\
\hline $\mathbf{H}$ & Algorithme de Marquardt \\
\hline
\end{tabular}

Par souci d'évaluation des performances du programme FAZO, il est apparu que les validations gagneraient à être élargies en comparant FAZO à d'autres logiciels, notés de $\mathrm{A}$ à $\mathrm{H}$ sur le tableau II, élaborés dans des laboratoires de recherche ayant participé à une campagne d'intercomparaison des techniques de déconvolution des spectres de thermoluminescence (GLOCANIN) (Bos et al., 
1993; Bos et al., 1994). Les procédures de minimisation ainsi que les équations cinétiques utilisées sont données dans les tableaux Il et III. Cette intercomparaison consistait à décomposer les spectres en leurs pics constituants et à déterminer les paramètres cinétiques $E$ et $s$ de chaque pic. Les résultats de FAZO sont comparés aux moyennes arithmétiques de l'ensemble des résultats pour chacun des paramètres $E$ et $s$ en ignorant, pour ce calcul, les résultats présentant d'importants écarts par rapport aux moyennes estimées. Des six spectres de l'intercomparaison, quatre sont présentés dans ce travail : GLOCANIN.1, figure 6 ( $a$ et b), et GLOCANIN.2, figure 7 ( $a$ et b).

TABLEAU III

Équations cinétiques des programmes A à $\mathrm{H}$ de l'intercomparaison GLOCANIN.

Kinetic equations of the programs $A$ to $H$ of the GLOCANIN intercomparison.

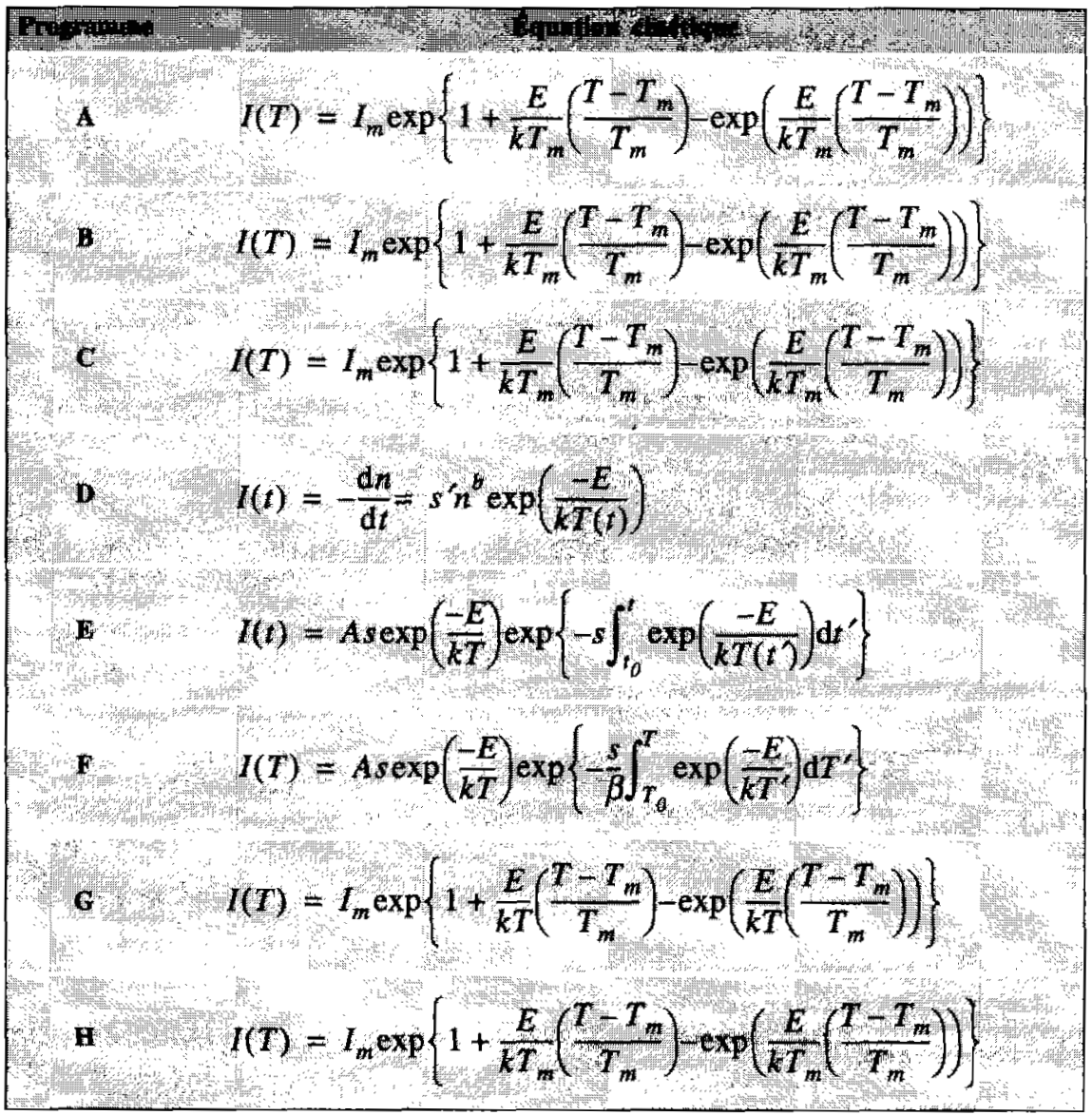



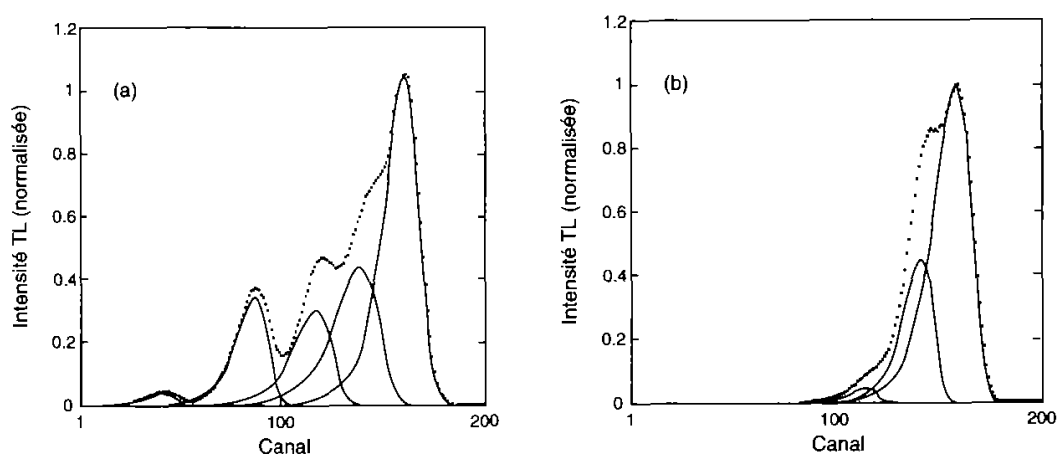

Fig. 6. - Déconvolution des spectres de l'intercomparaison GLOCANIN: (a) spectre GLOCANIN.I.a à 5 pics (pour $10 \mathrm{mGy}$ sans traitement thermique); (b) spectre GLOCANIN.1.b à 3 pics (pour $10 \mathrm{mGy}$ après traitement thermique).

Deconvolution of the GLOCANIN intercomparison glow curves: (a) GLOCANIN.I.a glow curve with 5 peaks (for 10 mGy without thermal treatment); (b) GLOCANIN.1.b glow curve with 3 peaks (for $10 \mathrm{mGy}$ after thermal treatment).
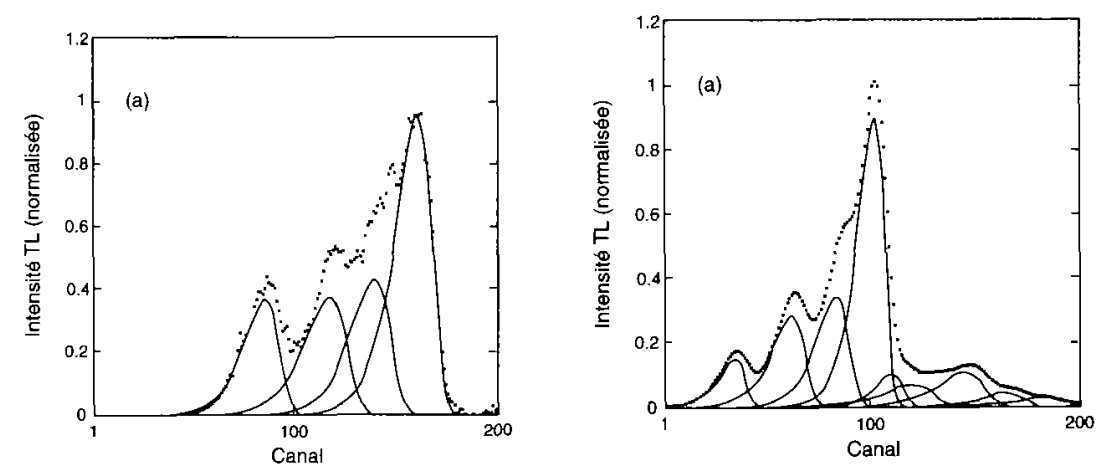

Fig. 7. - Déconvolution des spectres de l'intercomparaison GLOCANIN: (a) spectre GLOCANIN.2.a à 4 pics (pour $0.2 \mathrm{mGy}$ ); (b) spectre GLOCANIN.2.b à 9 pics (pour $60 \mathrm{~Gy})$.

Deconvolution of the GLOCANIN intercomparison glow curves: (a) GLOCANIN.2.a glow curve with 4 peaks (for $0.2 \mathrm{mGy}$ ); (b) GLOCANIN.2.b. glow curve with 9 peaks (for $60 \mathrm{~Gy})$.

Sur le tableau IV ( $a$ et $b$ ) et le tableau V ( $a$ et $b$ ) sont reportés les facteurs de mérite (FOM) ainsi que les paramètres cinétiques $E$ et $S$ pour chaque pic des quatre spectres. Il en ressort que les valeurs obtenues par FAZO sont très proches des valeurs moyennes. 


\section{TABLEAU IV}

Résultats des paramètres cinétiques $E$ et $s$ pour les spectres GLOCANIN.1.a et GLOCANIN.1.b obtenus par FAZO. ( $\bar{E}$ et $\bar{s}$ : moyennes respectifs de $E$ et $s$ des résultats des programmes $\mathrm{A}$ à $\mathrm{H} ; \Delta E$ et $\Delta \ln (s)$ écarts par rapport aux moyennes de $E$ et $\ln (s)$ ).

Kinetic parameters $E$ and $s$ results of the spectra GLOCANIN.1.a and GLOCANIN.1.b obtained by FAZO. ( $\bar{E}$ and $\bar{s}$ : respectively $E$ and $s$ means of the results of $A$ to $H$ programs; $\Delta E$ et $\Delta \ln (s)$ deviation from the means of $E$ et $\ln (s)$ ).

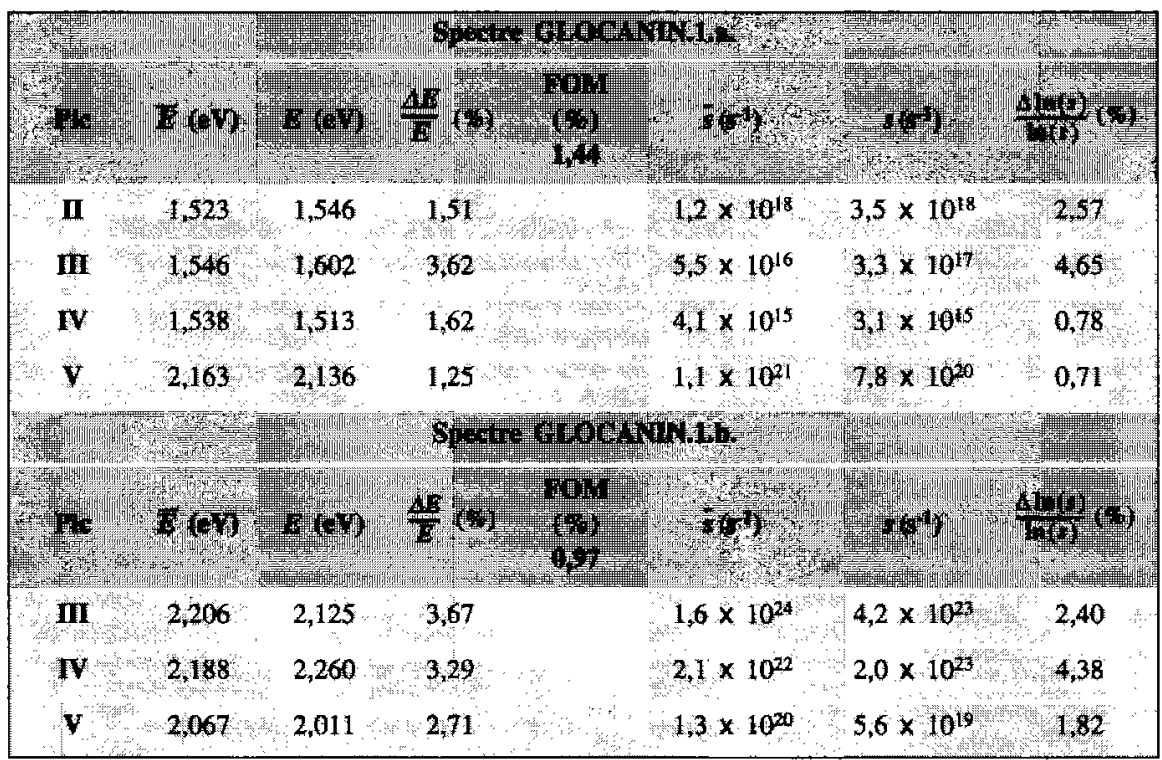

Un des aspects les plus intéressants qui est apparu au cours de ces validations, et qui mérite d'être signalé ici, porte sur la détermination des paramètres cinétiques du pic le plus stable, pic $\mathrm{V}$ ou pic dosimétrique, du détecteur LiF:Mg, Ti. En effet, il a été trouvé que les énergies d'activation $E$ et les facteurs de fréquence $s$, pour la plupart des spectres des détecteurs TLD 700 et TLD 100 déconvolués par FAZO, sont proches de $2 \mathrm{eV}$ et $10^{21} \mathrm{~s}^{-1}$. Ces valeurs sont en très bon accord avec celles publiées dans la littérature où $E$ est compris entre 2,06 et $2,2 \mathrm{eV}$ et $s$ entre $10^{19}$ et $10^{23} \mathrm{~s}^{-1}$ [17]. 
ANALYSE DES SPECTRES DE THERMOLUMINESCENCE

\section{TABLEAU V}

Résultats des paramètres cinétiques $E$ et $s$ pour les spectres GLOCANIN.2.a et GLOCANIN.2.b obtenus par FAZO.

Kinetic parameters $E$ and $s$ results of the spectra GLOCANIN.2.a and GLOCANIN.2.b obtained by FAZO.

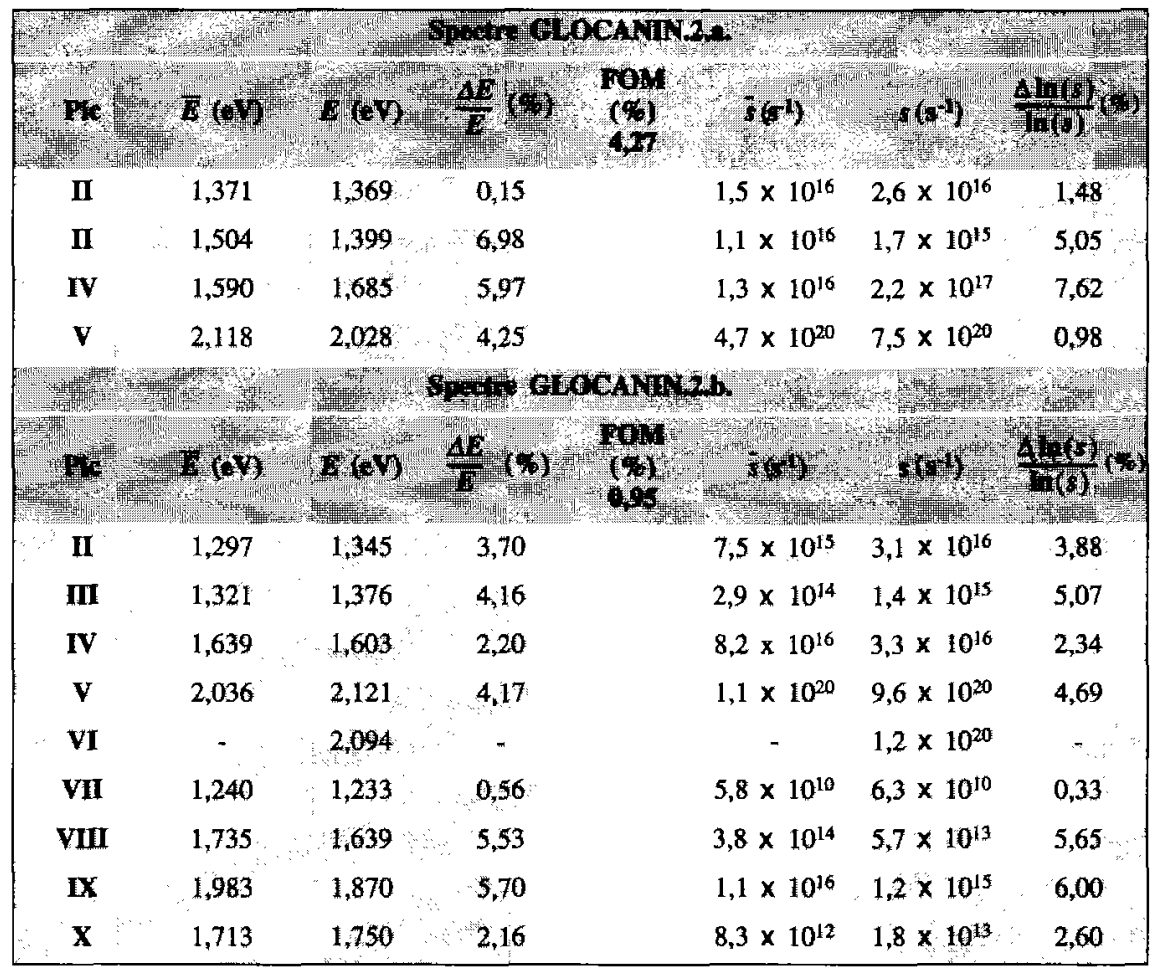

\section{Conclusion}

Il est admis depuis plusieurs années que la déconvolution des spectres de thermoluminescence permet l'amélioration de l'évaluation des doses ainsi que, dans certains cas, la discrimination des contributions des différents rayonnements dans les champs mixtes (neutrons- $\gamma$ ). La précision avec laquelle ces doses sont déterminées dépend de la puissance du programme de déconvolution utilisé.

Le programme FAZO élaboré au sein de notre laboratoire offre des performances intéressantes pour l'étude et l'analyse des spectres de thermoluminescence. Outre 
l'efficacité de FAZO pour la restitution des spectres avec des facteurs de mérite très satisfaisants, les paramètres cinétiques des pics déconvolués garantissent une évaluation exacte des doses d'exposition. Ces résultats sont d'un intérêt certain pour la dosimétrie individuelle et la mesure du rayonnement gamma ambiant en général, et l'étude des matériaux thermoluminescents en particulier.

Remerciements. Les auteurs tiennent à remercier Monsieur D.E. Cherouati, chef du département de la dosimétrie des rayonnements du CRS, pour ses précieuses remarques.

\section{Références}

Balian H., Eddy N.W. (1977) Figure Of Merit (FOM), an improved criterion over the normalized chisquared test for assessing goodness of fit of gamma-ray spectral peaks. Nucl. Inst. Meth., 145, 389-395.

Bos A.J.J., Piters T.M., Gomez Ros J.M., Delgado A. (1993) An intercomparison of glow curve analysis computer programs: I. Synthetic glow curves. Radiat. Prot. Dosim., 47, 473-477.

Bos A.J.J., Piters T.M., Gomez Ros J.M., Delgado A. (1994) An intercomparison of glow curve analysis computer programs : II. Measured glow curves. Radiat. Prot. Dosim., 51, 257-264.

Chen R. (1994) kinetics of thermoluminescence glow peaks. Dans : thermoluminescence and thermoluminescent dosimetry. (Horowitz Y.S., Eds) pp. 49-88. CRC Press, Florida.

Dehimi F.Z. (1995) Développement d'une Technique d'A nalyse des Spectres de Radiothermoluminescence par la Méthode de Déconvolution. Thèse de Magister, Centre de Radioprotection et de Sưreté. Alger.

Fletcher R. (1972) Methods for the solution of optimization problems. Comp. Phys. Comm., 3, 159-172.

Fletcher R. (1971) A modified Marquardt subroutine for non linear least squares. AERE-R.6799. Atomic Energy Research Establishment, Harwell.

HARSHAW/FLLTROL. (1988) Computerized glow curve deconvolution. User's Manual. HARSHAW/FILTROL. Ohio. U.S.A.

Jansson P.A. (1984) Deconvolution. With application in spectroscopy. Academic Press. New York.

Mc Keever S.W.S. (1985) Thermoluminescence analysis. Dans: Thermoluminescence of Solids. pp. 64-126. Cambridge University Press. Cambridge.

Mebhah D., Seghour F.Z. (1996) Amélioration des performances des détecteurs radiothermoluminescents. Rapport interne CRS/DOS/02/1996. Centre de Radioprotection et de Sûreté. Alger.

Nash J.C. (1990) Minimising a nonlinear sum of squares. Dans : Compact Numerical Methods for Computers, linear algebra and function minimisation pp. 207-217. Adain Hilger, Bristol.

Portal G. (1981) Preparation and properties of principal TL products. Dans : Applied Thermoluminescence Dosimetry. pp 97-122 M. Oberhofer and A. Scharmann, Eds Adam Hilger, Bristol.

Press W.H., Flannery B.P., Teukolsky S.A. and Vetterling W.T. (1988) Modeling of data. Dans : Numerical Recipes in C. pp. 517-565. Cambridge University Press. Cambridge.

Randall J.T., Wilkins J.T. (1945) The Phosphorescence of Varions Solids. Proc. R. Soc. London Ser. A., 184, 347.

Seghour F.Z. (1997) Guide d'utilisation du logiciel FAZO. Rapport interne CRS/DOS/01/1997. Centre de Radioprotection et de Sûreté. Alger.

Yossian D., Mahajna S., Ben Shachar B., Horowitz Y.S. (1993) Reinvestigation of the kinetics trapping parameters of peaks 5 in TLD-100 via "prompt" and "residual" isothermal decay. Radiat. Prot. Dosim., 47, 129-133. 